\title{
Virtual surgical planning, flow simulation, and 3-dimensional electrospinning of patient-specific grafts to optimize Fontan hemodynamics
}

Dominik Siallagan, MS, ${ }^{\mathrm{a}, \mathrm{b}}$ Yue-Hin Loke, MD, ${ }^{\mathrm{c}}$ Laura Olivieri, MD, ${ }^{\mathrm{a}, \mathrm{c}}$ Justin Opfermann, PhD, ${ }^{\mathrm{a}}$ Chin Siang Ong, MBBS, ${ }^{\mathrm{d}}$ Diane de Zélicourt, PhD, ${ }^{\mathrm{e}, \mathrm{f}}$ Anastasios Petrou, MS, ${ }^{\mathrm{b}}$ Marianne Schmid Daners, PhD, ${ }^{\mathrm{b}}$ Vartan Kurtcuoglu, ${ }^{\mathrm{e}}{ }^{\mathrm{f}}$ Mirko Meboldt, PhD, ${ }^{\mathrm{b}}$ Kevin Nelson, PhD, ${ }^{\mathrm{g}}$ Luca Vricella, MD, ${ }^{\mathrm{d}}$ Jed Johnson, $\mathrm{PhD},{ }^{\mathrm{g}}$ Narutoshi Hibino, MD, PhD,${ }^{\mathrm{d}}$ and Axel Krieger, $\mathrm{PhD}{ }^{\mathrm{a}, \mathrm{h}}$

\section{ABSTRACT}

Background: Despite advances in the Fontan procedure, there is an unmet clinical need for patient-specific graft designs that are optimized for variations in patient anatomy. The objective of this study is to design and produce patientspecific Fontan geometries, with the goal of improving hepatic flow distribution (HFD) and reducing power loss $\left(\mathrm{P}_{\text {loss }}\right)$, and manufacturing these designs by electrospinning.

Methods: Cardiac magnetic resonance imaging data from patients who previously underwent a Fontan procedure $(\mathrm{n}=2)$ was used to create 3 -dimensional models of their native Fontan geometry using standard image segmentation and geometry reconstruction software. For each patient, alternative designs were explored in silico, including tube-shaped and bifurcated conduits, and their performance in terms of $\mathrm{P}_{\text {loss }}$ and HFD probed by computational fluid dynamic (CFD) simulations. The best-performing options were then fabricated using electrospinning.

Results: CFD simulations showed that the bifurcated conduit improved HFD between the left and right pulmonary arteries, whereas both types of conduits reduced $\mathrm{P}_{\text {loss. }}$. In vitro testing with a flow-loop chamber supported the CFD results. The proposed designs were then successfully electrospun into tissue-engineered vascular grafts.

Conclusions: Our unique virtual cardiac surgery approach has the potential to improve the quality of surgery by manufacturing patient-specific designs before surgery, that are also optimized with balanced HFD and minimal $\mathrm{P}_{\text {loss }}$, based on refinement of commercially available options for image segmentation,

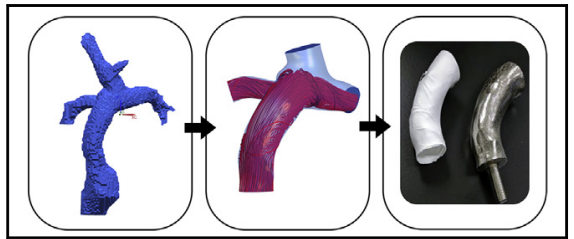

Virtual cardiac surgery with patient-specific graft design, optimization, and validation.

Central Message

Three-dimensional electrospinning has significant potential for implementing patient- specific solutions in the Fontan arena, allowing for the "printing" of grafts optimized in silico for hemodynamic performance.

\section{Perspective}

Ideal patient-specific conduit design is important for complex congenital cardiac surgery. Our integrated approach including image segmentation, computer-aided design based on flow simulations, patient-specific optimization, and manufacturing of a custom graft has the potential to improve the surgical result.

See Editorial Commentary page 1743. computer-aided design, and flow simulations. ( $\mathrm{J}$ Thorac Cardiovasc Surg 2018;155:1734-42)

The Fontan procedure is the critical, cumulative step in the overall surgical management of the single ventricle. The procedure ultimately results in a total cavopulmonary

From the ${ }^{\text {a Sheikh }}$ Zayed Institute for Surgical Innovation, Children's National Medical Center, Washington, DC; ${ }^{b}$ Product Development Group Zurich, Department of Mechanical and Process Engineering, ETH Zurich, Zurich, Switzerland; ${ }^{\mathrm{c}}$ Division of Cardiology, Children's National Health System, Washington, DC; ${ }^{\mathrm{d} D i v i s i o n}$ of Cardiac Surgery, Johns Hopkins Hospital, Baltimore, Md; ${ }^{\mathrm{e}}$ The Interface Group, Institute of Physiology, University of Zürich, Zürich, Switzerland; ${ }^{\text {SSwiss National }}$ Centre of Competence in Research, Kidney Control of Homeostasis, Zurich, Switzerland; ' Nanofiber Solutions, Inc, Hilliard, Ohio; and ${ }^{\mathrm{h}}$ Department of Mechanical Engineering, University of Maryland, College Park, Md.

This work was supported by the Eunice Kennedy Shriver National Institute of Child Health and Human Development, National Institutes of Health (Grant R21HD090671); the Swiss National Science Foundation (Grant 200021_147193 CINDY); and the Swiss National Centre of Competence in Research, Kidney Con- connection, such that the majority of systemic venous return passively enters the pulmonary arteries and bypasses the heart. ${ }^{1}$ Since its introduction in 1971, several modifications

trol of Homeostasis. The content is solely the responsibility of the authors and does not necessarily represent the official views of the National Institutes of Health.

Read at the 43rd Annual Meeting of the Western Thoracic Surgical Association, Colorado Springs, Colorado, June 21-24, 2017.

Received for publication July 5, 2017; revisions received Nov 9, 2017; accepted for publication Nov 27, 2017; available ahead of print Jan 17, 2018.

Address for reprints: Narutoshi Hibino, MD, PhD, Division of Cardiac Surgery, The

Johns Hopkins Hospital, Sheikh Zayed Tower, Ste 7107, 1800 Orleans St, Baltimore, MD 21287 (E-mail: nhibino1@jhmi.edu).

$0022-5223 / \$ 36.00$

Copyright (c) 2017 by The American Association for Thoracic Surgery

https://doi.org/10.1016/j.jtcvs.2017.11.068 


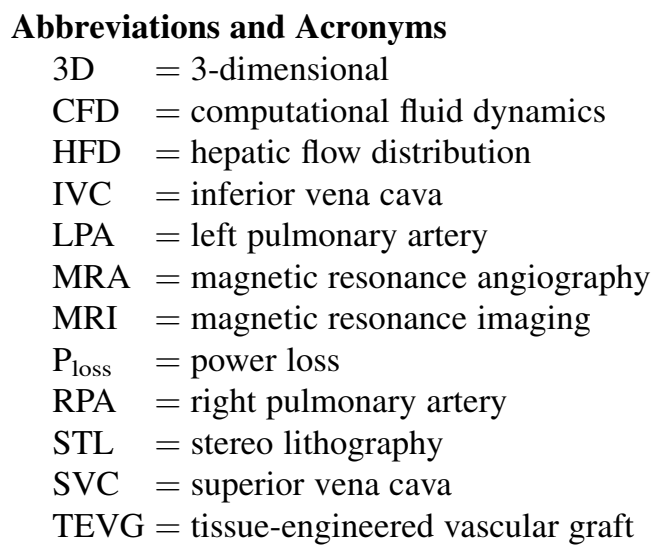

Scanning this $\mathrm{QR}$ code will take you to a supplemental video for the article.

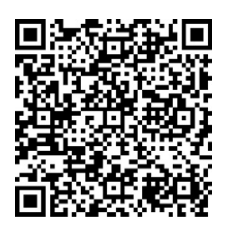

have been made to the procedure to improve hemodynamics and decrease the incidence of arrhythmia, including the intra-atrial tunnel, the extracardiac conduit, or more recently the intra/extracardiac modification ${ }^{2}$ or the bifurcated Y-graft design. ${ }^{3}$

Despite such advances in surgical technique and improvement in overall survival, patients with a Fontan circulation remain at risk for long-term complications, such as protein losing enteropathy, ventricular dysfunction, and arrhythmias. ${ }^{4}$ These patients also demonstrate poorer exercise performance and decreasing functional health status over time. ${ }^{5}$ The physiological mechanism for this decrease in exercise performance is multifactorial; from a fluid dynamics standpoint, power losses along narrowed vessels in the Fontan pathway have been shown to negatively affect exercise performance. $^{6,7}$ Thus, research into new Fontan conduit designs have focused on minimizing power loss $\left(\mathrm{P}_{\text {loss }}\right)$ to improve exercise capacity and potentially provide longterm benefits for patients' overall quality of life. Other hemodynamic elements can be optimized as well, such as balancing the hepatic flow distribution (HFD) across the pulmonary arteries, which may reduce the risk of pulmonary arteriovenous malformations. ${ }^{8}$ The use of simulation via computational fluid dynamics (CFD) has shown to be useful in proposing virtual patient-specific Fontan designs optimized for $\mathrm{P}_{\text {loss }}$ and $\mathrm{HFD}^{9,10}$; however, these virtual designs typically do not have commercially available counterparts for surgery, and thus this preoperative strategy cannot fully address the variable single-ventricle anatomy, for which there is no "one-size fits all" solution. ${ }^{11}$
Tissue-engineered vascular grafts (TEVGs) may serve as a bridge for implementing virtual designs. Three-dimensional custom-made TEVGs can be fabricated with a Food and Drug Administration-approved biodegradable nanofiber material composed of polyglycolic acid and poly-L-lactideco- $\varepsilon$-caprolactone electrospun along a 3D-printed mandrel. Our pre-clinical trial of TEVGs implanted into sheep models demonstrated remarkable resorption of the biodegradable scaffold over a 6-month period with development of an organized smooth muscle layer, extracellular matrix deposition, and endothelization; promisingly, the vascular grafts showed mechanical properties similar to those of native inferior vena cava (IVC). ${ }^{12}$ Thus, patient-specific grafts (whether bifurcated Y-grafts or extracardiac conduits) theoretically could be fabricated in this manner to produce an optimized Fontan pathway in vivo.

The objective of this study was to introduce a novel preoperative planning method for the Fontan procedure, where virtual patient-specific Fontan conduits are first optimized via CFD, and subsequently 3D manufactured into TEVGs via electrospinning (Video 1). This methodology could serve as a potential workflow to allow congenital heart surgeons to preoperatively visualize the Fontan circuit, optimize a conduit design, and ensure the preservation of simulated hemodynamics after the TEVGs are implanted in vivo.

\section{MATERIALS AND METHODS}

Magnetic Resonance Imaging and Magnetic

Resonance Angiography Flow Data Acquisition and

\section{Processing}

This study was approved by the Institutional Review Boards of all participating institutions. Cardiovascular magnetic resonance imaging (MRI) datasets from 2 patients who had previously undergone a Fontan surgery for the palliation of a single-ventricle heart defect were anonymized and exported in DICOM format. MRI data included contrast-enhanced, subtracted magnetic resonance angiography (MRA) and 4-phase velocity flow cines in the cavae and the pulmonary arteries. The MRA data, consisting of a late-phase, nongated, breath-held acquisition with pixel size $\sim 1.4 \times 1.4 \mathrm{~mm}$, served as a roadmap to build a 3-dimensional (3D) digital model of the Fontan circuit, including the proximal cavae and branch pulmonary arteries, using a
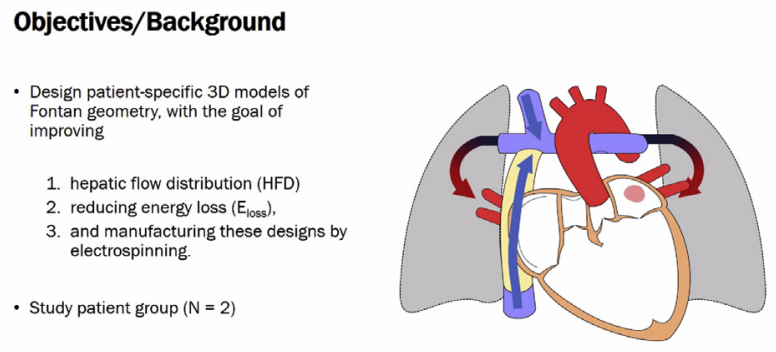

VIDEO 1. Video summarizing the methodology to create patient-specific Fontan grafts by using virtual surgical planning and 3D electrospinning. Video available at: http://www.jtcvsonline.org/article/S0022-5223(17) 32762-9/fulltext. 
commercially available image segmentation software (Mimics; Materialise, Leuven, Belgium). Both automatic thresholding and manual methods were used to identify the blood pool of the Fontan in each slice of the angiogram, allowing for the creation of a 3D digital Fontan model, which was then exported using the stereolithography (STL) file format. This STL file was hollowed and smoothed, and extensions were added to allow for velocity profiles to be partially developed at the anatomic inlets (superior vena cava [SVC] and IVC). Extensions were also added at the outlets (left pulmonary artery [LPA] and right pulmonary artery [RPA]) to avoid spurious influence of the boundary conditions in the domain of interest. Figure 1 illustrates the segmentation process, beginning with MRA and ending with the 3D digital model after the segmentation process.

Retrospectively-gated, through plane phase-encoded velocity maps were acquired across the IVC, SVC, LPA, and RPA using standard sequences, reconstructing 30 phases per cardiac cycle with a velocity encoding threshold of $150 \mathrm{~cm} / \mathrm{second}$. The time-averaged IVC and SVC flow rates were derived from the phase velocity data and prescribed as inlet boundary conditions to the CFD simulations. The time-averaged RPA and LPA flow rates were prescribed as outlet flow splits (the ratio of LPA to RPA). Finally, an additional sequence of phase velocity mapping was performed to measure the in-plane velocity along the coronal plane of the Fontan conduit to validate the flow structures predicted by CFD simulation.

\section{CFD Simulation}

CFD simulations were then performed to predict blood flow within the Fontan geometry, based on the inlet and outlet conditions extracted from MRI acquisition. The CFD simulation included several simplifications to ensure reasonable computational time while still capturing important fluid dynamic characteristics. Given the low Reynolds number, on the order of 900, the flow was modeled as laminar. The Fontan geometry was modeled with rigid walls, following a previously described procedure. ${ }^{3,13}$ Blood was modeled as an incompressible, Newtonian fluid with constant viscosity of $3.71 \mathrm{mPas}$ and a density of $1060 \mathrm{~kg} / \mathrm{m},{ }^{3,13}$ assumptions deemed acceptable in large vessels such as the pulmonary arteries and venae cavae.

IVC and SVC flows were prescribed to have a flat uniform flow profile with constant velocity, ${ }^{13}$ the extensions allowing for partial development before a total cavopulmonary connection. The corresponding velocity values of each inlet were derived from the MRI measurements averaged over one cardiac cycle. The outlet flow conditions were prescribed by the measured flow split ratio (LPA:RPA) of total inlet flow rate to maintain conservation of mass.
The commercial program Ansys FLUENT was used as the CFD simulation platform. The 3D unsteady Navier-Stokes equations were solved for 3000 time steps. The time step of each iteration was set at 0.001 seconds. Each step was solved within 30 iterations by the SIMPLE numerical solving method. The convergence values were set to $10^{-5}$ for $x, y, z$-velocity and mass conservation residuals.

The performance of the Fontan grafts, and optimization goals, were measured by 2 parameters: the $\mathrm{P}_{\text {loss }}$ across the Fontan geometry and HFD. $P_{\text {loss }}$ was calculated based on changes in the pressure and flow rates through each inlet and outlet, according to

$$
P_{\text {loss }}=\sum_{S V C, I V C} Q\left(\bar{p}+\frac{1}{2} \rho \cdot \bar{u}^{2}\right)-\sum_{R P A, L P A} Q\left(\bar{p}+\frac{1}{2} \rho \cdot \bar{u}^{2}\right)
$$

HFD was defined as the ratio of blood from the IVC to the LPA and RPA, respectively. The HFD was evaluated through particle tracking. A total of 3000 particles $\left(\mathrm{N}_{\text {tot }}\right)$ were seeded uniformly at the IVC inlet. The number of particles passing through the RPA and LPA outlets $\left(\mathrm{N}_{\mathrm{RPA}}\right.$ and $\mathrm{N}_{\mathrm{LPA}}$, respectively) was calculated at the end of the simulation using the velocity field at 3 seconds. The HFD was calculated according to

$$
\begin{aligned}
& H F D_{L P A}=\frac{N_{L P A}}{N_{t o t}} . \\
& H F D_{R P A}=\frac{N_{R P A}}{N_{t o t}}
\end{aligned}
$$

In vivo validation of simulation results was performed by comparison with MRI data, using the phase velocity map acquired along the coronal plane of the Fontan conduit. An equivalent simulated velocity map was first reconstructed and oriented along the same plane as the acquired MRI data. The velocity maps of simulation and MRI measurements were then compared, pixel-to-pixel, along 32 time measurements through the cardiac cycle. Velocity differences within $7.5 \mathrm{~cm} /$ second were considered acceptable.

\section{Graft Optimization}

Graft optimization aimed to minimize $\mathrm{P}_{\text {loss }}$ and maintain balanced HFD within the 60:40 range, similar to previous CFD studies. ${ }^{9}$ Two graft design strategies were investigated: tube-shaped conduits and bifurcated conduits. All graft designs were performed with the conventional computer-aided design program Solidworks (Dassault Systèmes, Vélizy-Villacoublay, France) using the Loft tool, which interpolates
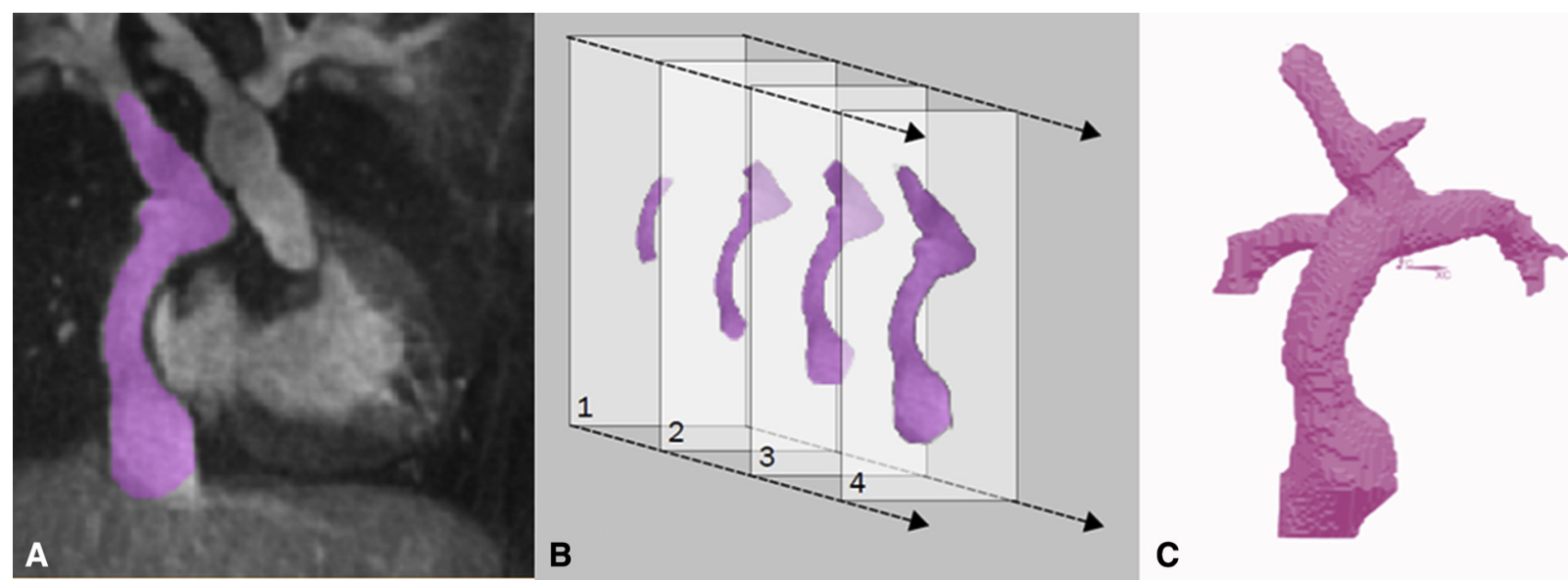

FIGURE 1. Starting with 3-dimensional (3D) contrast-enhanced magnetic resonance angiography (MRA) data, a 3D virtual model of the Fontan is built through a process called segmentation. A, The MRA with the region of interest, the Fontan blood pool, highlighted in purple. B, The Fontan highlighted in each slice of the MRA dataset. C, All of the slices combined to create a 3D virtual model. 
between 2 cross-sectional profiles along a defined curve. Different tube conduit variations were constructed from a fixed circular inlet profile (based on the native IVC area), a variable outlet profile, and a pathline. The bifurcated conduit design consisted of a stem with fixed inlet profile dividing into a left branch and a right branch of variable profiles.

For tube-shaped conduits, a manual strategy was implemented in an iterative fashion, first by exploring different locations for the insertion point within the feasible anastomoses area. Different outlet shapes were varied throughout the second iteration. In the third iteration, the size of the outlet was varied, followed by angle adjustments. After each iteration, the design with the best performance was used for the next iteration of adjustments and simulation (Figure 2). A 3D model of the surrounding heart and aorta anatomy was overlaid with the new graft designs, to minimize any overlap between the optimized graft and other thoracic features (eg, ascending aorta, vena cavae).

\section{In Vitro Testing}

Validation of the simulation results was performed by testing a 3D printed version of each conduit in a 4-chamber flow loop, based on an concept presented by Ochsner et al. ${ }^{14}$ The optimized conduits were first manufactured by exporting the conduit profile as an STL file and then
3D-printed using a Connex 3 Objet500 polyjet printer (Stratasys, Eden Prairie, Minn) with VeroWhite material. The printed conduits were connected to the experimental flow loop consisting of 4 chambers and 2 pumps, using a viscosity-matched glycerol-water mixture as a blood substitute The pumps were situated upstream of the inlets and set the flow of the SVC and IVC. Two pressure adjustable chambers, connected to the LPA and RPA, controlled the flow through the corresponding outlet. The pressures of each tank and the pumps were adjusted until the measured flows matched the in vivo time-averaged IVC and SVC flow rates and RPA:LPA flow splits. Pressure measurements were made during 3 independent trials for each of the retained designs. GraphPad Prism 7 (GraphPad Software, La Jolla, Calif) was used to analyze the data from the in vitro bench tests.

\section{Statistical Analysis}

To determine whether the data were parametric, a Shapiro-Wilk test for normality with $\alpha=0.05$ was performed on the in vitro pressure data. According to the Shapiro-Wilk test, the data did not differ significantly from a normal distribution $(P>.05)$, and so the data were assumed to be parametric. A 1 -sample $t$ test was used to determine if in vitro pressure data differed significantly from the theoretical mean as predicted by simulation. A 2 -sample unpaired $t$ test was used to

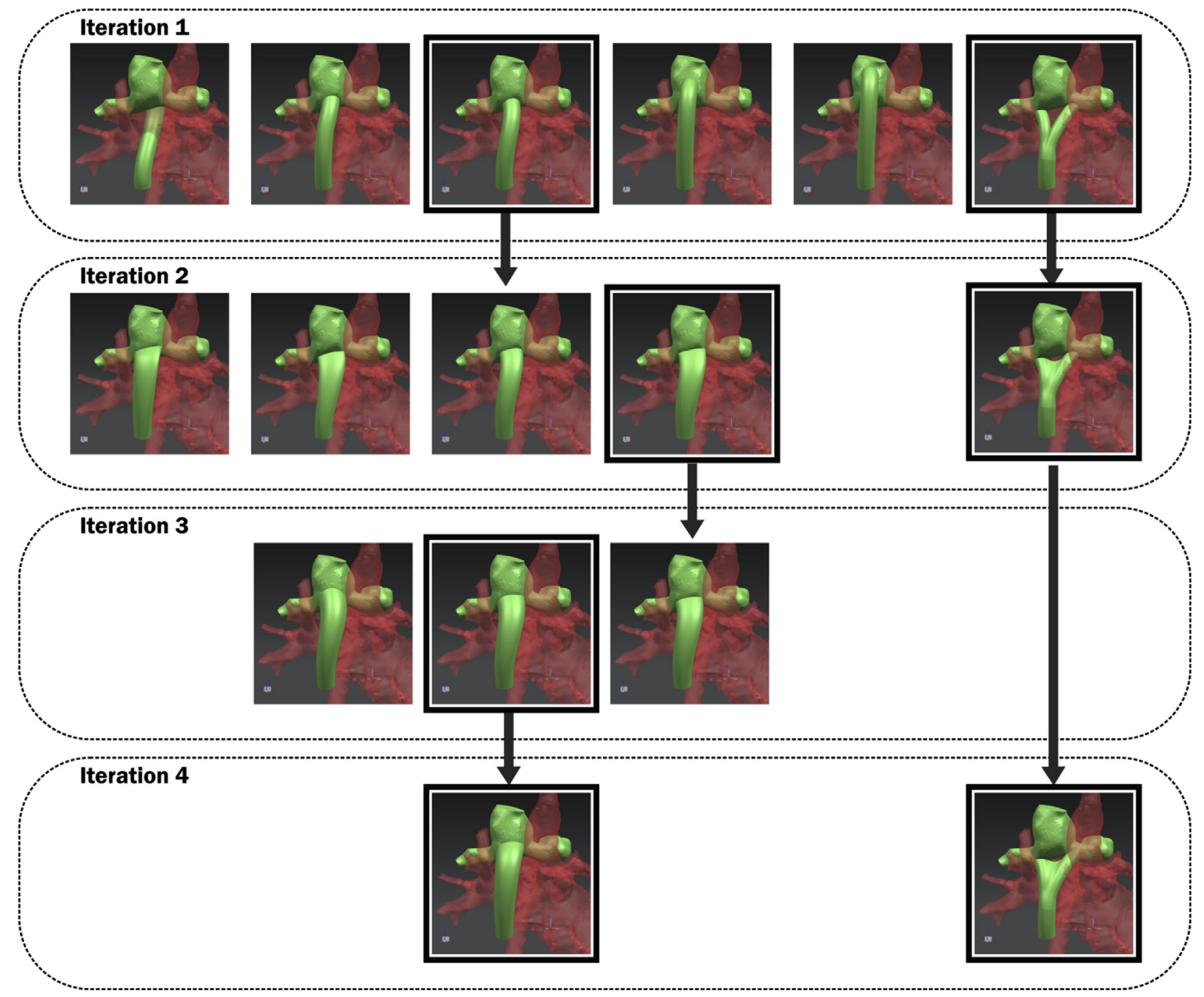

FIGURE 2. Diagram illustrating the optimization strategy with 4 iterations of adjustments. 

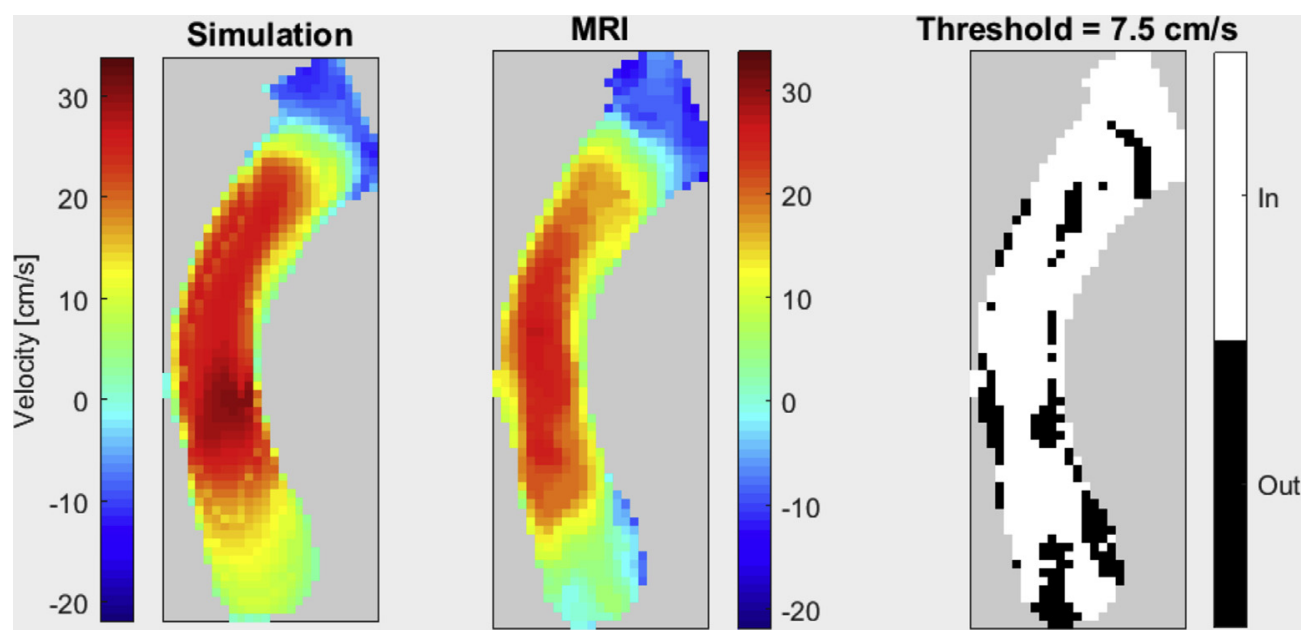

FIGURE 3. In vivo validation results comparing flow velocity profiles of simulated (left) and measured (middle) magnetic resonance imaging-detected flow. Subtracting the 2 shows that the areas of velocity difference are located primarily on the boundaries (right). MRI, Magnetic resonance imaging.

compare in vitro pressures between optimized and native conduit designs. The $t$ test was chosen because it is more robust to errors from small datasets, and our initial hypothesis considered only the relationships between measured data and the hypothetical mean. $P$ values are reported for pressures compared with their hypothetical mean, and for pressure comparisons between native and optimized graft designs. $P<.05$ was considered statistically significant for the Shapiro-Wilk and $t$ tests.

\section{Graft Manufacturing}

Once the optimized design was validated, a biodegradable version of each conduit was manufactured using a 3D electrospinning technique. A stainless steel mandrel in the shape of the optimized graft was 3D-printed by exporting the STL file of the graft to an external printing house (Shapeways, New York, NY). The mandrel was designed in such a way that the electrospun polymer graft could be removed. In case of the bifurcated grafts, the mandrel was a 3-part piece, pinned together, to allow it to be
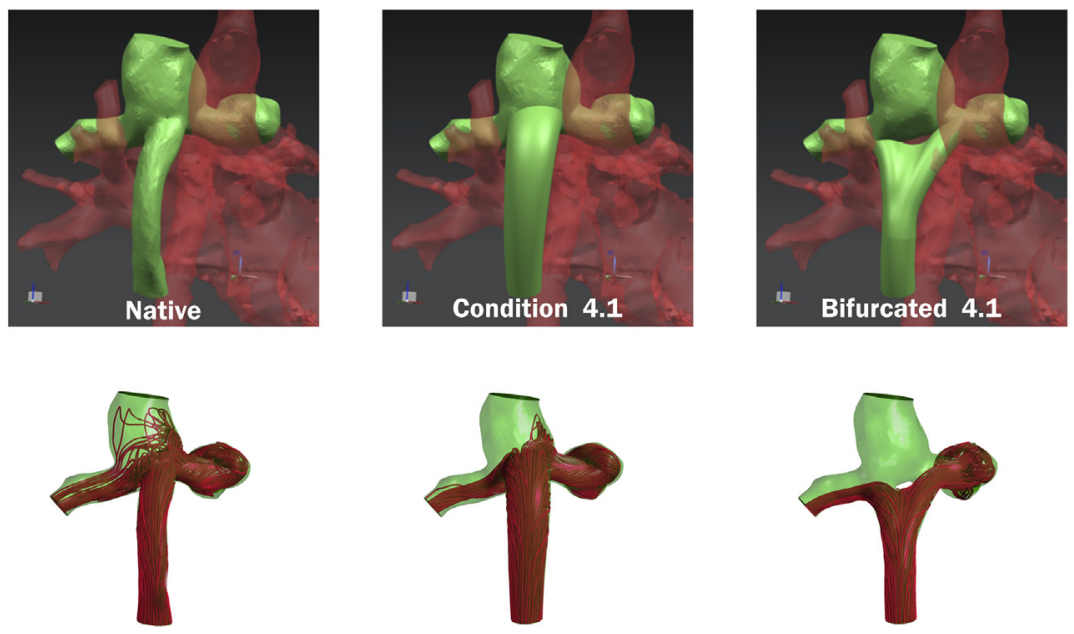

IVC to LPA [\%]
IVC to RPA [\%]
Power loss [mW]

73.8

25.5

57.4

40.8

42.1

58.8

4.52

3.42

2.89

FIGURE 4. Graft optimization results for patient 1. Native graft, best tube-like graft, and best bifurcated graft are shown with corresponding power loss and left/right pulmonary artery flow distribution. The tube is positioned in the center of the cavopulmonary anastomosis in a slightly anterior position, to avoid collision with the superior vena cava inflow. The outlet of the graft is elliptical-shaped to promote hepatic flow distribution. The area is $50 \%$ larger than the inlet area. The bifurcated graft is positioned in the center, and the branches are directed toward the pulmonary arteries. A 10-mm fillet is inserted in the middle of the branches to account for electrospinning manufacturers' specifications. IVC, Inferior vena cava; $L P A$, left pulmonary artery; $R P A$, right pulmonary artery. 
taken apart for graft removal. An additional pin was added to the mandrel to clamp it into the electrospinning setup.

Next, nano/micro polymer fibers were electrospun onto the mandrel (Nanofiber Solutions, Hilliard, Ohio). By applying high voltage to the polymer solution of a syringe and the mandrel, polymer fibers were deposited on the mandrel to create the graft design.

\section{RESULTS}

\section{In Vivo Validation of the CFD Simulations}

When reconstructed along the coronal plane of the Fontan conduit and compared with cardiac MRI phase contrast data, $75 \%$ of the simulated data remained within a representative threshold (Figure 3). Errors above the set threshold were found predominantly along the boundaries of the acquired velocity maps.

\section{Optimized Patient Designs}

Patient 1 (extracardiac Fontan). The results of 2 optimized designs are shown in Figure 4. The length of the tube-shaped conduit was similar to the native condition. The outlet profile had an elliptic shape (major axis, $12.4 \mathrm{~mm}$; minor axis, $9.6 \mathrm{~mm}$ ), and the insertion point was shifted approximately $5 \mathrm{~mm}$ toward the RPA. The insertion angle in the transverse plane was tilted approximately 35 degrees toward the RPA compared with the native condition. The insertion angle of the sagittal plane was tilted approximately 18 degrees toward the IVC. The stem of the bifurcated graft had a circular profile and was split into 2 branches with equal diameters of $11.3 \mathrm{~mm}$. The left branch was shifted $16 \mathrm{~mm}$ toward the lower side of the LPA, and the right branch was shifted $19 \mathrm{~mm}$ toward the RPA. The tube-shaped conduit and bifurcated graft designs showed improvements in HFD and $\mathrm{P}_{\text {loss }}$ compared with the native geometry. The tube-shaped conduit design demonstrated a $\mathrm{P}_{\text {loss }}$ of $3.42 \mathrm{~mW}$, a $24 \%$ decrease in $\mathrm{P}_{\text {loss }}$ compared with the native geometry. The HFD for LPA/ RPA also improved, from 74:26 to 57:42. The bifurcated graft also decreased $\mathrm{P}_{\text {loss }}$ by $38 \%$, to $2.89 \mathrm{~mW}$. The HFD improved to 41:59.

Patient 2 (intra-extracardiac Fontan). The geometrical limitations of this patient's anatomy-specifically, the positioning and size of the LPA-limited the optimization of a bifurcated graft design. The tube graft design relied on minor adjustments to positioning relative to the cavopulmonary anastomosis, resulting in an $11 \%$
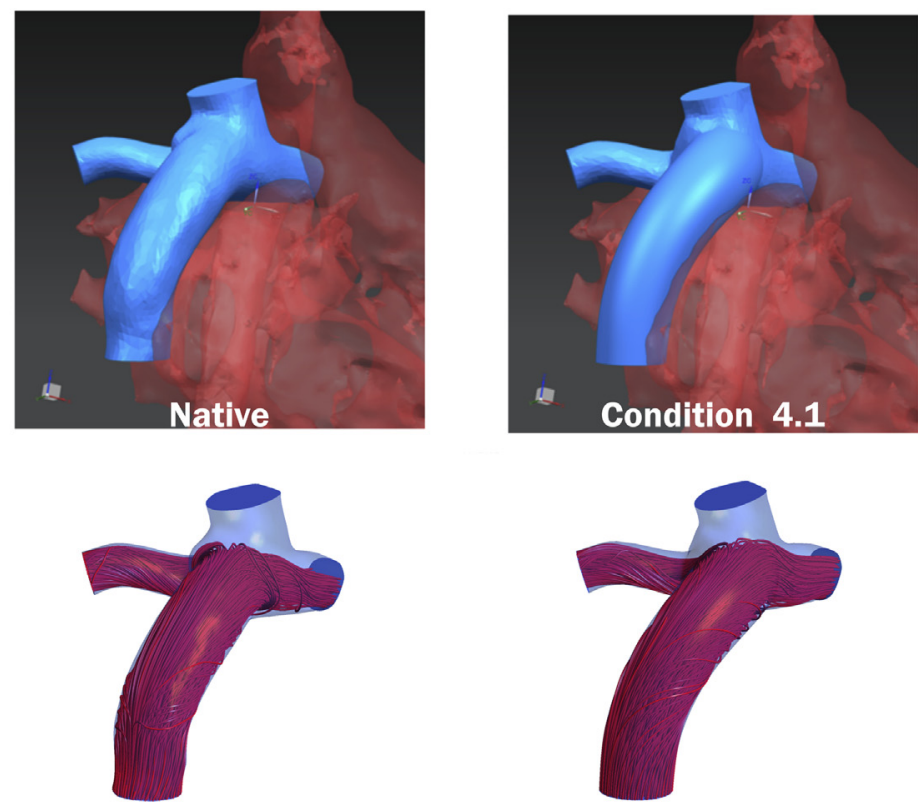

\section{IVC to LPA [\%] IVC to RPA [\%]

FIGURE 5. Graft optimization results for patient 2. Native graft and best tube-like graft are shown with corresponding power loss and left/right pulmonary artery flow distribution. Relative to the native geometry, the tube is positioned in the center of the cavopulmonary anastomosis and has a slightly anterior inflow direction to avoid direct flow competition with the superior vena cava inflow. The outlet of the graft has a circular shape with a radius of $10 \mathrm{~mm}$. IVC, Inferior vena cava; $L P A$, left pulmonary artery; $R P A$, right pulmonary artery. 
decrease in $P_{\text {loss }}$ (from 5.93 to $5.27 \mathrm{~mW}$ ) and a slight shift in HFD from 44:56 to 54:46 LPA:RPA (Figure 5). The outlet profile had a circular shape and was $3 \mathrm{~mm}$ larger in diameter than the inlet diameter. The insertion point was shifted approximately $6 \mathrm{~mm}$ toward the LPA. The insertion angle in the transverse plane was tilted approximately 40 degrees compared with the native condition. The insertion angle of the sagittal plane did not differ significantly.

\section{In Vitro Testing}

With the exception of the RPA pressure in native Fontan of patient $2(P=.004)$, the simulated pressures were in excellent agreement with the pressures measured in our in vitro test setup (Figure 6), demonstrating that our CFD simulations can accurately predict pressures and power losses in complex Fontan geometries, and can be applied toward the optimization of conduit geometries under set flow conditions. Maintaining caval and pulmonary flow constants, the reductions in $\mathrm{P}_{\text {loss }}$ observed in the optimized designs compared with the native ones reflect a reduction in Fontan pathway resistance. The optimized designs were thus associated with smaller pressure drops.

In our in vitro setup, all pressure changes were significant, except for LPA pressure in patient $2(P=.447)$. For patient 1, the pressure drops between the IVC and the SVC, LPA, and RPA were reduced by $0.296 \mathrm{~mm} \mathrm{Hg}$ $(P=.016), 0.284 \mathrm{~mm} \mathrm{Hg}(P=.039)$, and $0.247 \mathrm{~mm} \mathrm{Hg}$ $(P=.169)$, respectively, in the tube-shaped conduit and by $0.112 \mathrm{~mm} \mathrm{Hg}(P=.062), 0.270 \mathrm{~mm} \mathrm{Hg}(P=.024)$, and $0.232 \mathrm{~mm} \mathrm{Hg}(P=.170)$, respectively, in the bifurcated conduit. For patient 2, the pressure drops between the IVC and the SVC, LPA, and RPA were reduced by $0.073 \mathrm{~mm} \mathrm{Hg}(P=.141),-0.047 \mathrm{~mm} \mathrm{Hg}(P=.447)$, and $0.066 \mathrm{~mm} \mathrm{Hg}(P=.395)$, respectively, in the tubeshaped conduit. The pressure changes predicted by the CFD simulations were comparable to those measured in vitro for both patients.
Patient 1

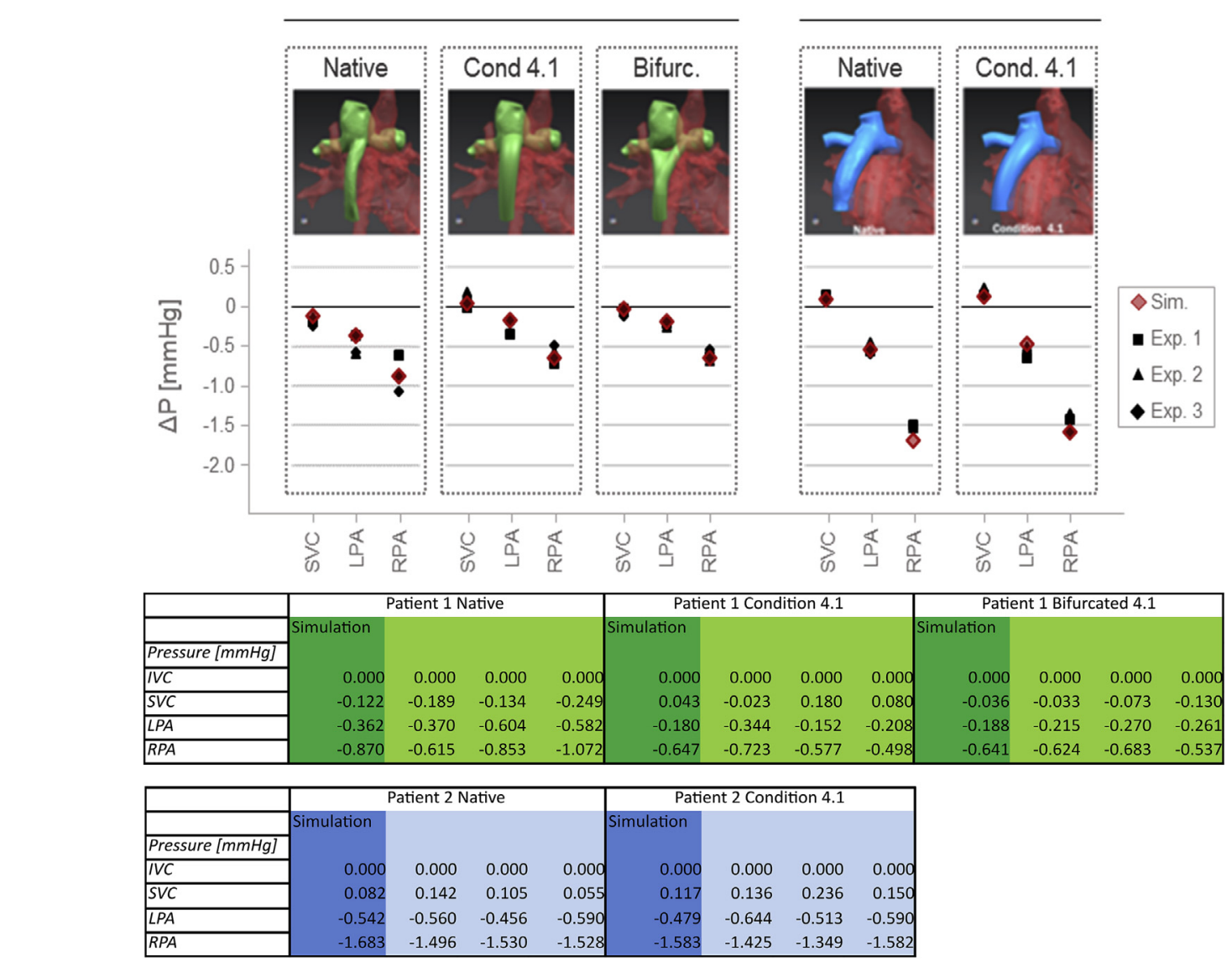

Patient 2

FIGURE 6. In vitro testing results to validate the computational fluid dynamics (CFD) simulation showing the experimental in vitro testing results in graphical form (top) and numerically in a table (bottom). Preliminary in vitro testing in a 4-chamber pressure- and flow-controlled test setup demonstrated a close match between CFD-simulated $(\mathrm{Sim})$ and in vitro-measured $(\mathrm{Nr} 1-3)$ pressures. $S V C$, Superior vena cava; $L P A$, left pulmonary artery; $R P A$, right pulmonary artery; $I V C$, inferior vena cava. 

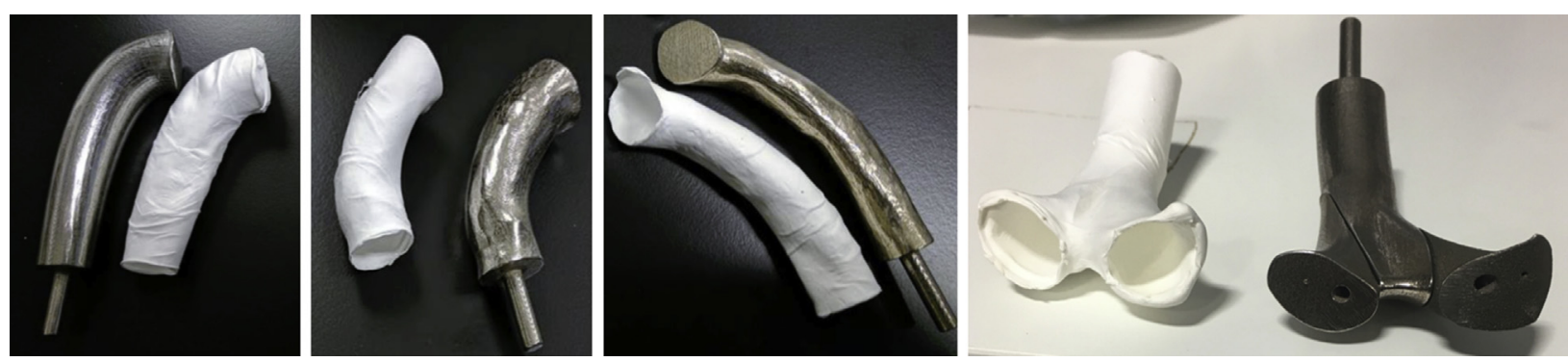

FIGURE 7. Pairs of 3-dimensional printed mandrels and electrospun biodegradable grafts (white). The pinned 3-part design of the mandrel for bifurcated grafts is shown at the right.

\section{Electrospun Optimized Grafts}

For the 2 patients, the native geometry, optimized tubeshaped grafts and bifurcated biodegradable grafts were successfully manufactured using electrospinning on 3D-printed stainless steel mandrels. The grafts easily detached from the mandrel, and in case of the bifurcated graft, the mandrel was disassembled using the pinned design before removal. Figure 7 shows pairs of manufactured electrospun vascular grafts and stainless steel mandrels.

\section{DISCUSSION}

Surgical management of the single-ventricle heart is a classic demonstration of the current unmet clinical need for customized, patient-specific grafts in congenital cardiac surgery. Although many surgical textbooks provide similar illustrations of how to perform the Fontan procedure, in reality, the significant variability in cardiac anatomy means that there is no true stereotypical approach to managing complex heart disease. Furthermore, using grafts of a standard shape without taking into account patient-specific anatomy may lead to an unequal distribution of hepatic blood flow across the LPA and RPA, with the formation of pulmonary arteriovenous malformations. ${ }^{15}$ A suboptimal Fontan geometry also leads to unnecessary $\mathrm{P}_{\text {loss }}$ and increased workload on the single-ventricle heart, hastening the onset of heart failure. ${ }^{16}$ In this regard, virtual surgery has enormous potential for designing graft with optimal geometry before congenital cardiac surgery ${ }^{17}$ and, specifically in our case, for improving HFD and reducing $\mathrm{P}_{\text {loss }}$ in Fontan patients, as shown in this study.

The use of CFD in the design of Fontan geometries has been reported previously. ${ }^{18,19}$ The key differences between previous work and our preset study include our use of an integrative and collaborative approach from image data acquisition to graft design and manufacturing, along with the use of in vitro testing to support our simulation data. In addition, the incorporation of TEVGs provided us with an increased degree of freedom for graft design. The integrated approach in particular was crucial in the translation of TEVGs into clinical application. We have previously demonstrated our ability to create patientspecific TEVGs using 3D printing and electrospinning technology for straight IVC conduits. ${ }^{12}$ In this report, we demonstrate the ability to manufacture TEVGs for patient-specific Fontan geometries as well. Our previous experience with electrospinning and the use of 3D-printed mandrels allowed us to account for the technical boundaries with the 3D printing technology and provided feedback regarding the feasibility of printing out certain conduit designs. The recognition of these boundaries provided us with useful feedback during the design of patient-specific Fontan conduits, in addition to the $\mathrm{P}_{\text {loss }}$ and HFD parameters measured.

Patient-specific 3D designs have been applied successfully in other surgical specialties, including orthopedic surgery, ${ }^{20}$ orthognathic surgery, and craniomaxillofacial surgery. ${ }^{21}$ The differences between these surgical fields and the cardiovascular field include (1) the need for suitable graft material with similar compliance as native vessels and biocompatibility with blood; (2) design optimization based on dynamic parameters, as opposed to static and relatively immobile boundary conditions; (3) and the need for consistent and accurate flow parameters provided by cardiac MRI sequences. Each represents a unique step and challenge in the use of 3D printing in the cardiovascular field. With an integrative and collaborative approach among the imaging clinicians, the engineers responsible for CFD and graft manufacturing, and the cardiovascular surgeon, these challenges can be addressed and overcome to bring patientspecific graft designs into the cardiovascular field.

One limitation of the present study is the small number of patients. In establishing this integrative approach, significant time and effort was spent developing an optimization strategy (Figure 2) and validating the CFD solver by in vivo and in vitro methods. We intend to apply this approach to other types of Fontan geometries. Despite the small number of patients, we observed that the benefit of custom-made versus standard grafts can differ between patients, which emphasizes the need for a patient-specific approach to identify the optimal strategy on an individual basis. In addition, our optimization strategy focused only on $\mathrm{P}_{\text {loss }}$ and HFD; surrogates for platelet activation as well as low flow regions and areas of low wall shear stress to assess the susceptibility to thrombosis will be included in 
future work. Future work also will include refining the simulation framework to take into account, for example, pulsatile flow and model exercise conditions, to further evaluate the significance of the results. We are planning to perform larger sets of in vitro flow loop comparisons with electrospun grafts to further validate our simulation results. We will also conduct mechanical testing of the patientspecific grafts for burst pressure and suture strength. Finally, there are currently no animal models suitable for a long-term Fontan study, and thus we lack long-term validation of the CFD via in vivo experiments with animal models. A feasible animal study would be constrained in terms of short-term outcomes, anastomosis results, and form retention properties of electrospun grafts. Therefore, future work will include large animal models with RV-PA venous structures to provide further preclinical supporting data before clinical application.

In conclusion, we have demonstrated a workflow allowing not only for the optimization of the Fontan pathway in silico, but also for the manufacture of the thus-optimized conduits. Although further computational, experimental, and animal testing is needed, this work provides a concrete first step toward making "virtual surgical planning" a reality.

\section{Conflict of Interest Statement}

Drs Johnson, Hibino, and Krieger are inventors listed on US Patent Application 62/209,990, which is related to this work. All other authors have nothing to disclose with regard to commercial support.

We thank Nanofiber Solutions, Inc for providing the graft materials used in this study.

\section{References}

1. John AS. Fontan repair of single-ventricle physiology: consequences of a unique physiology and possible treatment options. Cardiol Clin. 2015;33: 559-69.

2. Jonas RA. The intra/extracardiac conduit fenestrated Fontan. Semin Thorac Cardiovasc Surg Pediatr Card Surg Annu. 2011;14:11-8.

3. Marsden AL, Bernstein AJ, Reddy VM, Shadden SC, Spilker RL, Chan FP, et al. Evaluation of a novel Y-shaped extracardiac Fontan baffle using computational fluid dynamics. J Thorac Cardiovasc Surg. 2009;137:394-403.e2.

4. Pundi KN, Johnson JN, Dearani JA, Pundi KN, Li Z, Hinck CA, et al. 40-year follow-up after the Fontan operation: long-term outcomes of 1,052 patients. $J$ Am Coll Cardiol. 2015;66:1700-10.
5. Atz AM, Zak V, Mahony L, Uzark K, D'agincourt N, Goldberg DJ, et al. Longitudinal outcomes of patients with single ventricle after the Fontan procedure. $J$ Am Coll Cardiol. 2017;69:2735-44.

6. Khiabani RH, Whitehead KK, Han D, Restrepo M, Tang E, Bethel J, et al. Exercise capacity in single-ventricle patients after Fontan correlates with haemodynamic energy loss in TCPC. Heart. 2015;101:139-43.

7. Tang E, Wei ZA, Whitehead KK, Khiabani RH, Restrepo M, Mirabella L, et al. Effect of Fontan geometry on exercise haemodynamics and its potential implications. Heart. 2017;103:1806-12.

8. Pike NA, Vricella LA, Feinstein JA, Black MD, Reitz BA. Regression of severe pulmonary arteriovenous malformations after Fontan revision and "hepatic factor" rerouting. Ann Thorac Surg. 2004;78:697-9.

9. Yang W, Chan FP, Reddy VM, Marsden AL, Feinstein JA. Flow simulations and validation for the first cohort of patients undergoing the Y-graft Fontan procedure. J Thorac Cardiovasc Surg. 2015;149:247-55.

10. de Zélicourt DA, Kurtcuoglu V. Patient-specific surgical planning, where do we stand? The example of the Fontan procedure. Ann Biomed Eng. 2016;44: 174-86.

11. Yang W, Vignon-Clementel IE, Troianowski G, Reddy VM, Feinstein JA, Marsden AL. Hepatic blood flow distribution and performance in conventional and novel Y-graft Fontan geometries: a case series computational fluid dynamics study. J Thorac Cardiovasc Surg. 2012;143:1086-97.

12. Fukunishi T, Best CA, Sugiura T, Opfermann J, Ong CS, Shinoka T, et al. Preclinical study of patient-specific cell-free nanofiber tissue-engineered vascular grafts using 3-dimensional printing in a sheep model. J Thorac Cardiovasc Surg. 2017; 153:924-32.

13. de Zélicourt DA. Pulsatile Fontan Hemodynamics and Patient-Specific Surgical Planning: A Numerical Investigation. Atlanta GA: Georgia Institute of Technology; 2010.

14. Ochsner G, Amacher R, Amstutz A, Plass A, Schmid Daners M, Tevaearai H, et al. A novel interface for hybrid mock circulations to evaluate ventricular assist devices. IEEE Trans Biomed Eng. 2013;60:507-16.

15. Duncan BW, Desai S. Pulmonary arteriovenous malformations after cavopulmonary anastomosis. Ann Thorac Surg. 2003;76:1759-66.

16. Haggerty CM, Whitehead KK, Bethel J, Fogel MA, Yoganathan AP. Relationship of single ventricle filling and preload to total cavopulmonary connection hemodynamics. Ann Thorac Surg. 2015;99:911-7.

17. Ong CS, Loke YH, Opfermann J, Olivieri L, Vricella L, Krieger A, et al. Virtual surgery for conduit reconstruction of the right ventricular outflow tract. World $J$ Pediatr Congenit Heart Surg. 2017;8:391-3.

18. Tang E, Yoganathan AP. Optimizing hepatic flow distribution with the Fontan Ygraft: lessons from computational simulations. J Thorac Cardiovasc Surg. 2015; 149:255-6.

19. Yang W, Feinstein JA, Shadden SC, Vignon-Clementel IE, Marsden AL. Optimization of a Y-graft design for improved hepatic flow distribution in the Fontan circulation. J Biomech Eng. 2013;135:011002.

20. Hoang D, Perrault D, Stevanovic M, Ghiassi A. Surgical applications of threedimensional printing: a review of the current literature \& how to get started. Ann Transl Med. 2016;4:456.

21. Jacobs CA, Lin AY. A new classification of three-dimensional printing technologies: systematic review of three-dimensional printing for patient-specific craniomaxillofacial surgery. Plast Reconstr Surg. 2017;139:1211-20.

Key Words: 3D printing, flow dynamics, patient specific model, virtual surgical planning 